九州大学学術情報リポジトリ

Kyushu University Institutional Repository

\title{
A new method to calculate a 2D Ising universality transition point: Application near the Ashkin-Teller multicritical point
}

Moriya, Shunji

Department of Physics, Kyushu University

Nomura, Kiyohide

Department of Physics, Kyushu University

http://hdl. handle. net/2324/4481533

出版情報: Journal of the Physical Society of Japan. 89 (9), pp.093001-1-093001-4, 2020-08-28. 日本物理学会

バージョン：

権利関係 : ๑2020 The Physical Society of Japan 


\title{
A new method to calculate a 2D Ising universality transition point : Application near the Ashkin-Teller multicritical point
}

\author{
Shunji Moriya $^{1 *}$, and Kiyohide Nomura ${ }^{1}$ \\ ${ }^{1}$ Department of Physics, Kyushu University, Fukuoka 819-0395, Japan
}

\begin{abstract}
We propose a new method to numerically calculate transition points that belongs to 2D Ising universality class for quantum spin models. Generally, near the multicritical point, in conventional methods, a finite size correction becomes very large. To suppress the effect of the multicritical point, we use a $\mathrm{z}$-axis twisted boundary condition and a y-axis twisted boundary condition. We apply our method to an $S=\frac{1}{2}$ bond-alternating XXZ model. The multicritical point of this model has a BKT transition, where the correlation length diverges singularly. However, with our method, the convergence of calculation is highly improved, thus we can calculate the transition point even near the multicritical point.
\end{abstract}

Critical phenomena are one of the important subjects in condensed matter physics. As a typical solvable model, a classical 2D Ising model is studied widely. ${ }^{1)}$ In the some limit, the transfer matrix of a classical 2D Ising model becomes a quantum 1D Transverse-Field Ising (TFI) model. ${ }^{2) 3)}$ Several methods are proposed to calculate the 2D Ising universality transition points of quantum spin models. ${ }^{4) 5)}$ But, when the model has a multicritical point, the scaling behaviors become difficult due to the effect of multiple critical lines. So, conventional methods are not useful near a multicritical point.

Another method to calculate a transition point, a Level Spectroscopy (LS) method is useful to cancel logarithmic corrections of a Berezinskii-Kosterlitz-Thouless (BKT) transition. ${ }^{6-9)}$ But, the LS method can not be applied to 2D Ising universality transitions.

In this letter, as an example that has a multicritical point, we study an $S=\frac{1}{2}$ bond-alternating (BA) XXZ chain,

$$
\hat{H}=\sum_{j}^{L}\left[1-(-1)^{j} \delta\right]\left(\hat{S}_{j}^{x} \hat{S}_{j+1}^{x}+\hat{S}_{j}^{y} \hat{S}_{j+1}^{y}+\Delta \hat{S}_{j}^{z} \hat{S}_{j+1}^{z}\right),
$$

where $L=2 n$ ( $n$ is integer). This model is equivalent to the Ashkin-Teller model. ${ }^{11-13)}$ This Hamiltonian with periodic boundary condition $\left(\hat{\boldsymbol{S}}_{L+1}^{x}=\hat{\boldsymbol{S}}_{1}^{x}\right)$ is invariant under spin rotation around the z-axis $\left(\hat{U}_{\theta}^{z}=\exp \left(i \theta \sum_{j} \hat{S}_{j}^{z}\right)\right)$, spin reversal $\left(\hat{U}_{\pi}^{y}=\exp \left(i \pi \sum_{j} \hat{S}_{j}^{y}\right)\right)$ and two-sites translation $\left(\left(\hat{T}_{R}\right)^{2}\right.$ : $\hat{T}_{R}$ is one-site translation, $\left.\hat{T}_{R} \hat{\boldsymbol{S}}_{j} \hat{T}_{R}^{-1}=\hat{\boldsymbol{S}}_{j+1}\right)$. Corresponding eigenvalues are $M=\sum_{j} S_{j}^{z}, U_{\pi}^{y}= \pm 1, q=4 \pi n / L$ $(n=0, \cdots, L / 2-1)$. In addition, this model is symmetric about the sign of $\delta$, since the operation $\delta \rightarrow-\delta$ can be regarded as one-site translation $\hat{T}_{R} \hat{H} \hat{T}_{R}^{-1}$. At zero temperature, in the phase diagram of this model (1), the Gaussian universality transition line bifurcates into the two 2D Ising universality transition lines at $(\Delta=1, \delta=0)$ (Fig. 1). In Dimer1 phase $(\delta>0)$, the $2 j-1,2 j$ spins take a singlet pairing, and in Dimer2 phase $(\delta<0)$, the $2 j, 2 j+1$ spins take a singlet pairing, $(j=1,2, \cdots, L)$. In both phases, there exits a nondegenerate ground state with an energy gap. In Néel phase, the ground states are doubly degenerate and the spin reversal symmetry is broken. At the multicritical point $(\Delta=1, \delta=0)$,

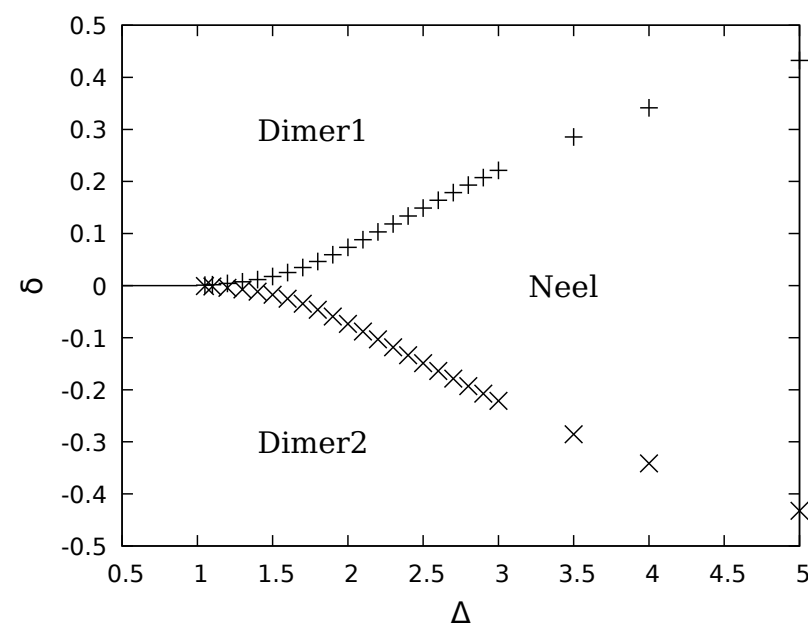

Fig. 1. Phase diagram in the $\Delta-\delta$ plane. Dimer1-Dimer2 phase boundary is the Gaussian universality and Dimer-Néel phase boundaries are the 2D Ising universality. We draw the 2D Ising universality transition lines by using the $\mathrm{L}=24$ numerical result of $\mathrm{yTBC}-\mathrm{zTBC}$ method, noted by + and $\times$.

called Ashkin-Teller multicritical point (AT point), a BKT transition occurs along $\delta=0$ where the correlation length diverges singularly. Near the AT point in $\Delta>1$, the two 2D Ising universality transition lines become extremely close. Thus, since the finite size correction terms become very large, one can not precisely calculate transition points near the AT point with the conventional methods. In our new method, getting an idea from the Kramers-Wannier duality, ${ }^{10)}$ we use the two boundary conditions (BC's), which enable to calculate the 2D Ising universality transition points very accurately even near the AT point

Anisotropic limit - We review that the BA XXZ model (1) is identical to the TFI model in the anisotropic limit, ${ }^{14)}$ then we shall discuss the boundary conditions. Firstly, we start in PBC. We separate Eq.(1) to even bond and odd bond,

$$
\hat{H}=\beta \sum_{j}^{L / 2}\left(\hat{S}_{2 j}^{x} \hat{S}_{2 j+1}^{x}+\hat{S}_{2 j}^{y} \hat{S}_{2 j+1}^{y}+\Delta \hat{S}_{2 j}^{z} \hat{S}_{2 j+1}^{z}\right)
$$




$$
+\sum_{j}^{L / 2}\left(\hat{S}_{2 j-1}^{x} \hat{S}_{2 j}^{x}+\hat{S}_{2 j-1}^{y} \hat{S}_{2 j}^{y}+\Delta \hat{S}_{2 j-1}^{z} \hat{S}_{2 j}^{z}\right),
$$

$\beta=\frac{1-\delta}{1+\delta}$ and ignore the constant factor of the Hamiltonian. In $\Delta \rightarrow \infty, \beta \rightarrow 0, \Delta \beta \sim O(1)$ limit, the $\Delta \hat{S}_{2 j-1}^{z} \hat{S}_{2 j}^{z}$ terms mostly contributes to the ground state energy. The ground state space is spanned by only

$$
\begin{aligned}
& \left|\uparrow_{2 j-1} \downarrow_{2 j}\right\rangle=\left|\uparrow_{j}\right\rangle^{\prime}, \\
& \left|\downarrow_{2 j-1} \uparrow_{2 j}\right\rangle=\left|\downarrow_{j}\right\rangle^{\prime},
\end{aligned}
$$

which are regarded as effective Ising spin states. The effective states and operators are denoted by '. The first and second terms $\hat{S}_{2 j}^{x} \hat{S}_{2 j+1}^{x}+\hat{S}_{2 j}^{y} \hat{S}_{2 j+1}^{y}$ vanish by $\beta \rightarrow 0$. The perturbative Hamiltonian is

$$
\begin{aligned}
& \hat{H}_{1}=\sum_{j}^{L / 2}\left(\beta \Delta \hat{S}_{2 j}^{z} \hat{S}_{2 j+1}^{z}\right)+\sum_{j}^{L / 2}\left(\hat{S}_{2 j-1}^{x} \hat{S}_{2 j}^{x}+\hat{S}_{2 j-1}^{y} \hat{S}_{2 j}^{y}\right) \\
& =\sum_{j}^{L / 2}\left(\beta \Delta \hat{S}_{2 j}^{z} \hat{S}_{2 j+1}^{z}\right)+\frac{1}{2} \sum_{j}^{L / 2}\left(\hat{S}_{2 j-1}^{+} \hat{S}_{2 j}^{-}+\hat{S}_{2 j-1}^{-} \hat{S}_{2 j}^{+}\right) .
\end{aligned}
$$

We consider the first order degenerate perturbation. The first terms of (4) are operating as

$$
\begin{array}{r}
\hat{S}_{2 j+1}^{z}\left|\uparrow_{j+1}\right\rangle^{\prime}=\frac{1}{2}\left|\uparrow_{j+1}\right\rangle^{\prime}, \\
\hat{S}_{2 j+1}^{z}\left|\downarrow_{j+1}\right\rangle^{\prime}=-\frac{1}{2}\left|\downarrow_{j+1}\right\rangle^{\prime}, \\
\hat{S}_{2 j}^{z}\left|\uparrow_{j}\right\rangle^{\prime}=-\frac{1}{2}\left|\uparrow_{j}\right\rangle^{\prime}, \\
\hat{S}_{2 j}^{z}\left|\downarrow_{j}\right\rangle^{\prime}=\frac{1}{2}\left|\downarrow_{j}\right\rangle^{\prime} .
\end{array}
$$

$\hat{S}_{2 j}^{z} \hat{S}_{2 j+1}^{z}$ can be regarded in the effective space as $-\hat{S}^{\prime}{ }_{j}^{z} \hat{S}^{\prime}{ }_{j+1}$. The second and third terms are operating as

$$
\begin{aligned}
& \hat{S}_{2 j-1}^{+} \hat{S}_{2 j}^{-}\left|\downarrow_{j}\right\rangle^{\prime}=\left|\uparrow_{j}\right\rangle^{\prime}, \\
& \hat{S}_{2 j-1}^{-} \hat{S}_{2 j}^{+}\left|\uparrow_{j}\right\rangle^{\prime}=\left|\downarrow_{j}\right\rangle^{\prime} .
\end{aligned}
$$

Thus, $\frac{1}{2}\left(\hat{S}_{2 j-1}^{+} \hat{S}_{2 j}^{-}+\hat{S}_{2 j-1}^{-} \hat{S}_{2 j}^{+}\right)$can be regarded in the effective space as $\frac{1}{2}\left(\hat{S}^{\prime}{ }_{j}^{+}+{\hat{S^{\prime}}}_{j}{ }_{j}\right)={\hat{S^{\prime}}}_{j}{ }_{j}$. In summary, the effective Hamiltonian becomes

$$
\hat{H}^{\prime}=\sum_{j}^{L / 2}\left(-\beta \Delta \hat{S}^{\prime}{ }_{j}{\hat{S^{\prime}}}^{z}{ }_{j+1}+{\hat{S^{\prime}}}_{j}{ }_{j}\right) .
$$

By operating $\exp \left(i \pi \sum_{i}^{L / 2} \hat{S}_{j}{ }_{j}^{z}\right)$, the effective Hamiltonian becomes the TFI model,

$$
\hat{H}^{\prime}=\beta \Delta \sum_{j}^{L / 2}\left(-\hat{S}^{\prime}{ }_{j} \hat{S}^{\prime}{ }_{j+1}^{z}-\gamma \hat{S}^{\prime}{ }_{j}^{x}\right) \quad\left(\gamma \equiv \frac{1}{\beta \Delta}\right),
$$

that has an order-disorder transition at $\gamma=1$, because of the Kramers-Wannier duality. ${ }^{10)}$ This Hamiltonian is invariant under spin reversal and one-site translation. We rewrite the Hamiltonian (6), taking account of the boudary condition,

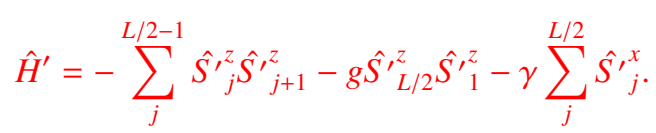

From exact solution, ${ }^{15)}$ for a finite system size $L, E_{0}(L, g=$ $\left.1, U_{\pi}^{y}=-1\right)=E_{0}\left(L, g=-1, U_{\pi}^{y}=1\right)+2(\gamma-1)$ is satisfied. $E_{0}$ means a lowest state energy. At the transition point $\gamma=1$,
Table I. Quantum numbers of the eigenstates of PBC, zTBC and yTBC for $\delta>0$. The case for $\delta<0$ is denoted by (). The states used for yTBC-zTBC method are denoted with $\bullet$ and yTBC-PBC method with $\circ$.

\begin{tabular}{|c|c|c|}
\hline & $M$ & $U_{\pi}^{y}$ \\
\hline$\circ \mathrm{PBC}$ & 0 & $-1(-1)$ \\
\hline$\bullet \mathrm{zTBC}$ & 0 & $-1(1)$ \\
\hline$\bullet$ yTBC & even (odd) & $1(1)$ \\
\hline
\end{tabular}

the energies on the two BC's are crossing,

$$
E_{0}\left(L, g=1, U_{\pi}^{y}=-1\right)=E_{0}\left(L, g=-1, U_{\pi}^{y}=1\right) .
$$

We can determinate the transition point of the TFI model by Eq. (8).

Next, we discuss what BC's of the BA XXZ model are corresponding to the $g=1,-1$ of the TFI model. For the BA XXZ model, we introduce z-axis twisted BC (zTBC),

$$
S_{L+1}^{x}=-S_{1}^{x}, S_{L+1}^{y}=-S_{1}^{y}, S_{L+1}^{z}=S_{1}^{z} .
$$

The zTBC conserves the spin rotational symmetry and the spin reversal symmetry, but breaks the two-sites translational symmetry. In the anisotropic limit, the effective Hamiltonian becomes the $g=1$ TFI model, since the $\mathrm{x}, \mathrm{y}$-direction boundary terms vanish.

And, we introduce y-axis twisted BC (yTBC),

$$
S_{L+1}^{x}=-S_{1}^{x}, S_{L+1}^{y}=S_{1}^{y}, S_{L+1}^{z}=-S_{1}^{z} .
$$

The yTBC (10) conserves the spin reversal symmetry, but breaks the two-sites translational symmetry and the spin rotational symmetry. About the last point, since the boundary terms become

$$
-\hat{S}_{L}^{x} \hat{S}_{1}^{x}+\hat{S}_{L}^{y} \hat{S}_{1}^{y}-\Delta \hat{S}_{L}^{z} \hat{S}_{1}^{z}=-\frac{1}{2}\left(\hat{S}_{L}^{+} \hat{S}_{1}^{+}+\hat{S}_{L}^{-} \hat{S}_{1}^{-}\right)-\Delta \hat{S}_{L}^{z} \hat{S}_{1}^{z},
$$

thus $M$ can not be conserved. But, the Hamiltonian is particularly invariant under $\pi$-rotation around z-axis $\hat{U}_{\pi}^{z}=$ $\exp \left(i \pi \sum_{j} \hat{S}_{j}^{z}\right)=(-1)^{\hat{M}}$. So, a parity of a total magnetization $P_{M}=(-1)^{M}$ is a conserved quantity. In the anisotropic limit, the z-direction boundary terms remain minus, $-\Delta \hat{S}_{L}^{z} \hat{S}_{1}^{z}$. So, the effective Hamiltonian becomes the $g=-1$ TFI model.

Consequently, PBC and zTBC of the BA XXZ model correspond to $g=1$ of the TFI model, and the yTBC corresponds to $g=-1$. Thus, Eq. (8) of the TFI model is extended to the BA XXZ model,

$$
E_{0}^{P B C}\left(M=0, U_{\pi}^{y}=-1\right)=E_{0}^{y T B C}\left(M=\text { even, } U_{\pi}^{y}=1\right),
$$

(hereafter we call the yTBC-PBC method) or

$$
E_{0}^{z T B C}\left(M=0, U_{\pi}^{y}=-1\right)=E_{0}^{y T B C}\left(M=\text { even, } U_{\pi}^{y}=1\right),
$$

(we call the yTBC-zTBC method). The above quantum numbers are for $\delta>0$. In Table I, the quantum number are summarized for $\delta>0$ and $\delta<0$. Although the finite size corrections vanish in the anisotropic limit, they remain in the finite $\Delta$ region because of a perturbation in the process from Eq. (2) to Eq. (7). However, we consider that the relation of Eq. (11) and (12) are supported by the 2D Ising universality class or the $c=1 / 2$ conformal field theory. We shall discuss on this point in a future paper.

Isotropic limit - On the self dual line $(\delta=0)$, the Hamilto- 


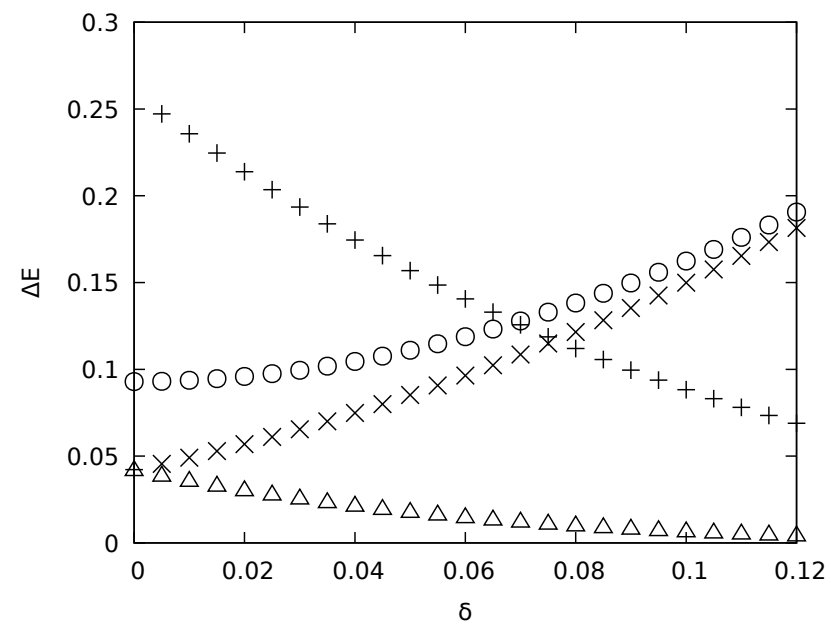

Fig. 2. The energies of each $\mathrm{BC}$ for $\mathrm{L}=14$. The value of $\Delta$ is fixed at 2.0 and $\delta$ is changed. $\bigcirc$ is $E_{0}^{P B C}\left(M=0, U_{\pi}^{y}=-1\right), \times$ is $E_{0}^{z T B C}\left(M=0, U_{\pi}^{y}=-1\right)$, $\triangle$ is $E_{0}^{z T B C}\left(M=0, U_{\pi}^{y}=1\right),+$ is $E_{0}^{y T B C}\left(M=\right.$ even, $\left.U_{\pi}^{y}=1\right)$. The PBC lowest energy $E_{0}^{P B C}\left(M=0, U_{\pi}^{y}=1\right)$ is subtracted from each energy.

nian with zTBC becomes,

$$
\begin{array}{r}
\hat{H}=\sum_{j}^{L-1}\left(\hat{S}_{j}^{x} \hat{S}_{j+1}^{x}+\hat{S}_{j}^{y} \hat{S}_{j+1}^{y}+\Delta \hat{S}_{j}^{z} \hat{S}_{j+1}^{z}\right) \\
-\hat{S}_{L}^{x} \hat{S}_{1}^{x}-\hat{S}_{L}^{y} \hat{S}_{1}^{y}+\Delta \hat{S}_{L}^{z} \hat{S}_{1}^{z} .
\end{array}
$$

We define $\pi$ spin rotation at $j$ site about $z$-axis as $\hat{u}_{j}^{z}=$ $\exp \left(i \pi \hat{S}_{j}^{z}\right)$. Since the Hamiltonian (13) is invariant under $\hat{T}_{R} \hat{u}_{L}^{z}$,

$$
\hat{T}_{R} \hat{u}_{L}^{z} \hat{H}\left|U_{\pi}^{y}\right\rangle=\hat{H} \hat{T}_{R} \hat{u}_{L}^{z}\left|U_{\pi}^{y}\right\rangle .
$$

The commutation relation between $\hat{U}_{\pi}^{y}$ and $\hat{u}_{L}^{z}$ is

$$
\begin{aligned}
\hat{U}_{\pi}^{y} \hat{u}_{L}^{z} & =\exp \left(i \pi \sum_{j} \hat{S}_{j}^{y}\right) \exp \left(i \pi \hat{S}_{L}^{z}\right) \\
& =\exp \left(-i \pi \hat{S}_{L}^{z}\right) \exp \left(i \pi \sum_{j} \hat{S}_{j}^{y}\right) \\
& =\exp \left(-2 i \pi \hat{S}_{L}^{z}\right) \hat{u}_{L}^{z} \hat{U}_{\pi}^{y} .
\end{aligned}
$$

When $S$ is a half-integer, the eigenvalue of $\hat{S}_{L}^{z}$ is a half-integer,

$$
\hat{U}_{\pi}^{y} \hat{u}_{L}^{z}=-\hat{u}_{L}^{z} \hat{U}_{\pi}^{y}
$$

So,

$$
\begin{aligned}
\hat{U}_{\pi}^{y} \hat{T}_{R} \hat{u}_{L}^{z}\left|U_{\pi}^{y}=1\right\rangle & =-\hat{T}_{R} \hat{u}_{L}^{z} \hat{U}_{\pi}^{y}\left|U_{\pi}^{y}=1\right\rangle \\
& =-\hat{T}_{R} \hat{u}_{L}^{z}\left|U_{\pi}^{y}=1\right\rangle \\
& \equiv-\left|U_{\pi}^{y}=-1\right\rangle .
\end{aligned}
$$

Consequently, $\left|U_{\pi}^{y}=1\right\rangle$ and $\left|U_{\pi}^{y}=-1\right\rangle$ are degenerate for an arbitrary $L$,

$$
E_{0}^{z T B C}\left(L, M=0, U_{\pi}^{y}=-1\right)=E_{0}^{z T B C}\left(L, M=0, U_{\pi}^{y}=1\right),
$$

as you can see in Fig.2 at $\delta=0$. Furthermore, on the isotropic point $(\Delta=1)$, the yTBC is equivalent to zTBC. Thus, replacing zTBC of the right side of Eq. (18) with yTBC,

$$
E_{0}^{z T B C}\left(L, M=0, U_{\pi}^{y}=-1\right)=E_{0}^{y T B C}\left(L, M=\text { even, } U_{\pi}^{y}=1\right) .
$$
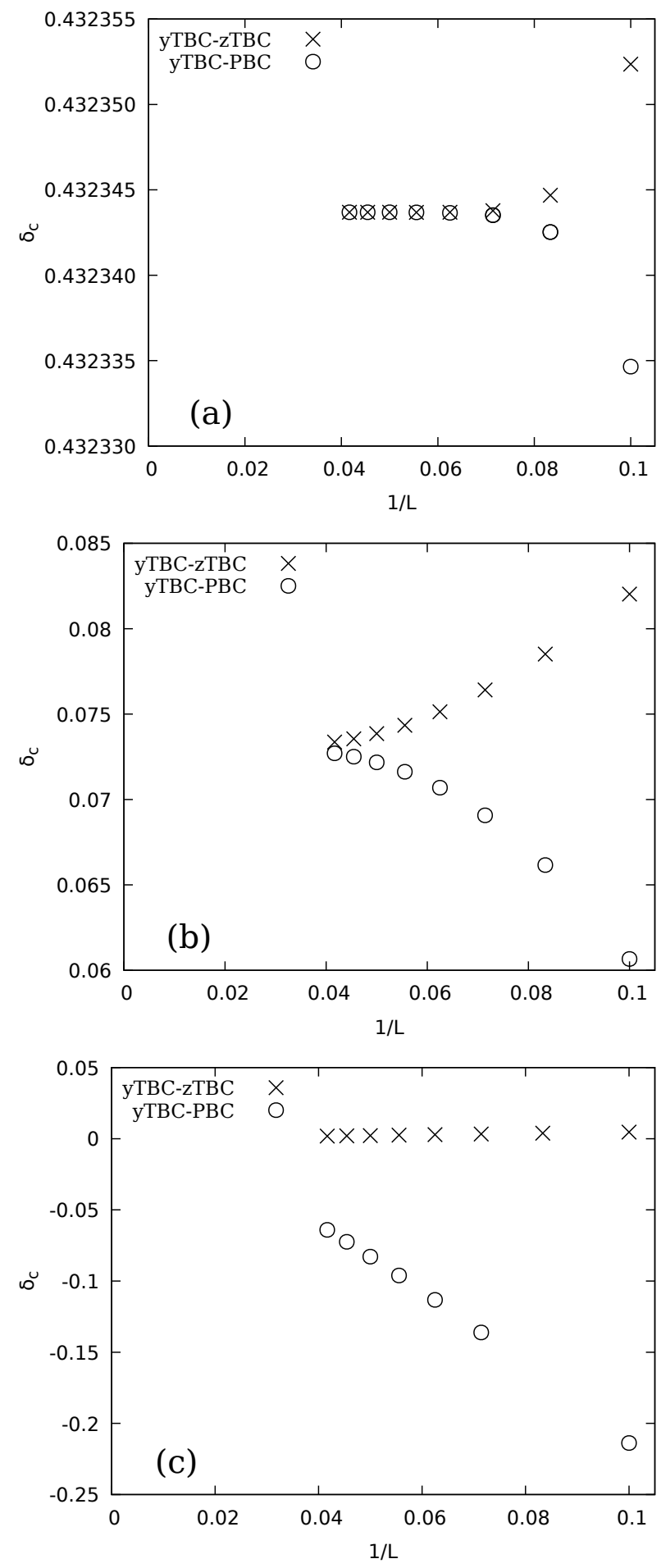

Fig. 3. The size dependence of the crossing point $\delta_{c}$ of the yTBC-РBC method (O) and the yTBC-zTBC method ( $\times$ ), (a) in $\Delta=5.0$, (b) in $\Delta=2.0$, (c) in $\Delta=1.1$.

Consequently, since the correction terms vanish at the AT point, the yTBC-zTBC method (12) can suppress the effect of the AT point. In contrast, the correction terms of the yTBCPBC method remains at the AT point.

Numerical calculation - We use the exact diagonalization method to calculate the energies of each BC. We determine the energy crossing point $\delta_{c}$, by fixing $\Delta$ and changing $\delta$, on $\mathrm{L}=10, \cdots, 24$, as Fig.2. Note that the PBC ground state 


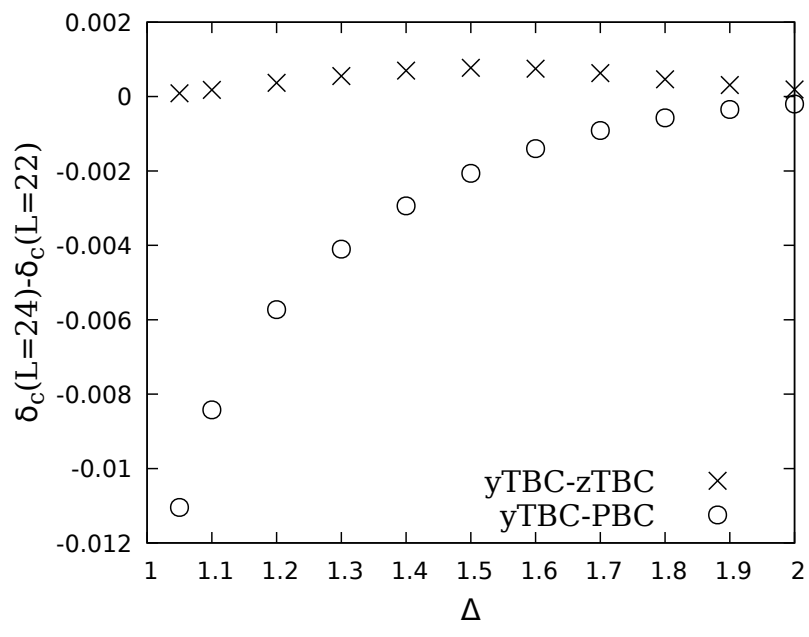

Fig. 4. The size difference of the crossing point $\delta_{c} \mathrm{~L}=24$ and $\mathrm{L}=22$. $\bigcirc$ is the yTBC-PBC method, $\times$ is the yTBC-zTBC method.

and the zTBC lowest state are not related with the KramersWannier duality (8). We show the phase diagram Fig.1. The size dependence shows in Fig. 3. The crossing points $\delta_{c}(L)$ by the yTBC-zTBC method are monotonically decreasing with size L, whereas those of the yTBC-PBC method are monotonically increasing. As Fig. 3(a), in the large $\Delta$ region, the yTBC-zTBC method and the yTBC-PBC method are rapidly converged. Next, near the AT point, as Fig. 3(c), the finite size corrections of the yTBC-PBC method become very large. In contrast, the yTBC-zTBC method shows a well convergence.

To compare the two methods from another viewpoint, we show the size difference of the crossing point $\delta_{c}(L=24)-$ $\delta_{c}(L=22)$ in Fig. 4. For large $\Delta$, the two methods are almost the same and the size difference vanish. Near the AT point, the size difference of the yTBC-PBC method becomes very large, but the yTBC-zTBC method approaching to zero.

Conclusion - Using the yTBC-PBC method (11) or the
yTBC-zTBC method (12), we can numerically calculate 2D Ising universality transition points. We actually calculate the transition lines of $\mathrm{S}=1 / 2 \mathrm{BA} X X Z$ model. As expected, the yTBC-zTBC method reduces the finite size effects near the multicritical point, since the finite size correction terms vanish at AT point. About critical exponents and the universality class, we shall describe them in a future paper. Furthermore, we verify the accuracy by comparing the yTBC-zTBC numerical result with the result of renormalization group theory. ${ }^{17)}$ We expect our method can be applied to several quantum spin models.

Acknowledgement - Our calculation program used in the exact diagonalization is TITPACK Ver. 2 coded by H. Nishimori. ${ }^{16)}$ A modification of the calculation program for yTBC is assisted by T.Mukai.

1) L.Onsager, Phys.Rev, 65,117 (1944).

2) E.H.Lieb, T.D.Schultz, and D.C.Mattis, Ann. Phys. (N.Y.), 16,407 (1961).

3) P.Pfeuty, Ann. of Phys, 57,79 (1970).

4) H.Nishimori, K.Okamoto and M. Yokozawa, J. Phys. Soc. Jpn, 56, 4126 (1987).

5) Tzeng YC, Dai L, Chung MC, et al. Sci Rep 6, 26453 (2016).

6) A. Kitazawa, J. Phys. A30, L285 (1997).

7) K. Nomura and A. Kitazawa, J. Phys. A.: Math. Gen. 31, 7341 (1998).

8) K.Nomura and A.Kitazawa, J. Phys. Soc. Jpn 66, 12, (1997).

9) A. Kitazawa and K. Nomura, J. Phys. Soc. Jpn 66, 11, (1997).

10) H. A. Kramers and G. H. Wannier, Phys. Rev. 60, 252 (1941).

11) J. Ashkin and E. Teller, Phys. Rev. 64, 178 (1943).

12) C. Fan, Phys. Lett. A 39, 136 (1972).

13) M.Kohmoto, M. den Nijs, and L. P. Kadanoff, Phys. Rev. B 24, 5229 (1981).

14) M.Yamanaka, Y.Hatsugai, and M.Kohmoto. Phys. Rev. B48, 9555, (1993).

15) G.Cabrera and R.Jullien. Phys. Rev. B35, 7062 (1987).

16) H.Nishimori and Y.Taguchi. Prog. Theor. Phys. Suppl, 87247 (1986).

17) L.P.Kadanoff, Phys. Rev. B22, 1405, (1980). 